\title{
i-collaboration 3.0: um framework de apoio ao desenvolvimento de Ambientes Distribuídos de Aprendizagem Sensíveis ao Contexto
}

\author{
Eduardo A. Oliveira ${ }^{1}$, Patrícia Tedesco ${ }^{1}$ \\ ${ }^{1}$ Centro de Informática - Universidade Federal de Pernambuco (UFPE) \\ Caixa Postal 7851 - 50.732-970 - Recife - PE - Brasil \\ \{eao, pcart\} @cin.ufpe.br
}

\begin{abstract}
As a way of encouraging the distributed and context-sensitive learning in the Web, as well as keeping the students interacting, sharing knowledge and motivated, this work proposes the i-collaboration 3.0. The framework seeks, through the use of an intelligent agent, to support interoperability between different Web environments, to enable the consolidation of distributed data of students in these different environments, as well as to customize learning content. To verify that the framework was indeed interesting, an experiment was carried with Social Web Learning system support, developed over the proposed framework. The results indicated that the framework contributed to the promotion of learning among students.
\end{abstract}

Resumo. Como forma de incentivar o aprendizado distribuído e sensível a contexto na Internet, neste trabalho propõe-se o framework $i$-collaboration 3.0. O framework busca, por meio do uso de um agente inteligente, apoiar a interoperabilidade entre diferentes ambientes Web, a consolidação de dados distribuídos de estudantes nestes diferentes ambientes, a personalização e a adaptação de conteúdos de aprendizagem e, a sensibilidade a contexto. Para verificar se o framework era de fato interessante, foi realizado um experimento a partir do Sistema Social Web Learning, desenvolvido sobre o framework proposto. Os resultados indicaram que o framework contribuiu para a promoção do aprendizado entre os estudantes envolvidos.

\section{Introdução}

Diferente do que acontecia há alguns anos atrás, o aluno que estuda a distância hoje está muito mais familiarizado com os recursos da internet (em suas várias plataformas e redes sociais, um fenômeno conhecido como nomadismo digital (Meyrowitz, 2004)) e está acostumado a compartilhar ideias e trocar experiências em grupo. Com esta mudança de paradigma, fica ainda mais difícil de fazer os estudantes de cursos virtuais se interessarem por um Ambiente Virtual de Aprendizagem (AVA) tradicional, onde muitas vezes as interfaces não estão adequadas às suas necessidades e, principalmente, muitas vezes as ferramentas colaborativas não permitem que este aluno estabeleça relações com os demais colegas, a exemplo do que acontece naturalmente nas redes sociais. As redes sociais, sites, blogs e outras plataformas Web têm evoluído e se reinventado muito mais rapidamente do que os AVA. Os estudantes não percebem os avanços nos AVA na mesma velocidade com que percebem mudanças nas redes sociais, 
por exemplo. O AVA acompanha a evolução lenta e natural da educação tradicional; ele não tem se reinventado na mesma velocidade que a internet, de modo a atender as necessidades dos novos estudantes digitais, os estudantes do século 21. Muitos AVA têm se apresentado de maneira semelhante ao longo dos anos (de maneira generalizada, possuem um repositório para objetos de aprendizagem e algumas ferramentas colaborativas) e continuam lidando com todos os estudantes da mesma maneira, de modo impessoal, sem atender as necessidades particulares de cada um (Oliveira e Tedesco, 2009).

Além destes problemas, diversos outros são encontrados nos AVA atuais, a exemplo da falta de interoperabilidade/integração entre AVA e seus conteúdos, dificuldades para criar e reutilizar informações contidas nos AVA, buscas não inteligentes/personalizadas de conteúdos, dentre outros; estes problemas também contribuem para a desmotivação de estudantes que utilizam AVA. O contexto dos estudantes nem sempre é levado em consideração no processo de aprendizagem. Contexto é o que está por trás da habilidade de definir o que é ou não relevante em um dado momento, e, neste caso em particular permite que o AVA filtre e dissemine informações mais úteis e adapte seus serviços às necessidades particulares do estudante, provendo recomendações e, adaptações em interfaces (para que fiquem mais flexíveis e fáceis de usar) (Vieira et al, 2006). Além da falta de contexto em grande parte dos AVA, devido ao conteúdo do curso virtual estar centralizado em um único AVA, de maneira isolada e não muito atrativa e amigável como acontece nas redes sociais, quando poderia estar acessível de maneira distribuída e personalizada no meio virtual, os estudantes não se sentem atraídos e motivados para buscar a informação nestes ambientes. As redes sociais, os blogs e as ferramentas de comunicação instantânea - Skype e GTalk, não têm sido totalmente exploradas como um veículo útil para a construção do conhecimento humano, individual e/ou coletivo.

Considerando que a Internet possui potencial em recursos de comunicação e interação, informações armazenadas e possibilidades para diversas combinações, seja de recursos, informações e/ou pessoas, no que tange o crescimento do conhecimento humano, o desafio de permitir que os estudantes possam aprender de maneira distribuída e sensível ao contexto motivou a seguinte questão neste trabalho:

- Como prover um ambiente de aprendizagem distribuído e personalizado com os recursos existentes na Web?

A partir da questão que motivou esta pesquisa, outras duas perguntas foram relacionadas ao problema: (i) Como compartilhar, ao mesmo tempo, o conhecimento de um curso virtual de maneira descentralizada nas redes sociais, em AVA e em sites na Internet? (ii) Como adaptar e personalizar conteúdos de aprendizagem de um determinado curso virtual para atender as necessidades particulares de cada estudante nos mais diversos ambientes da Internet onde o curso se encontra disponível?

A hipótese que conduziu este trabalho foi: Se os estudantes da EAD puderem acessar os conteúdos de seus cursos virtuais de forma personalizada e adequada ao contexto e, de maneira distribuída a partir dos ambientes Web a que estão habituados, então eles se sentirão incentivados a interagir mais nestes ambientes para buscar conteúdos de seus cursos virtuais.

Com base na hipótese formulada, o objetivo geral deste trabalho foi propor um framework de apoio a criação de ambientes virtuais distribuídos de aprendizagem 
sensíveis ao contexto. Os ambientes virtuais de aprendizagem distribuídos e sensíveis ao contexto permitirão que os estudantes acessem os conteúdos de aprendizagem de seus cursos a partir dos ambientes Web que frequentam habitualmente. Estes conteúdos devem ainda ser acessados de maneira personalizada e adaptada, para atender as necessidades particulares de cada estudante.

Um sistema (Social Web Learning - SWL) foi desenvolvido sobre o framework proposto e, com o uso deste sistema, foi realizado um experimento durante 2 meses com alunos do primeiro período do curso de ciência da computação da Universidade Católica de Pernambuco (UNICAP) para validação da hipótese formulada. Os resultados obtidos após o experimento indicam que o Sistema SWL atendeu às expectativas dos estudantes. Após 2 meses de utilização do sistema, observamos o satisfatório interesse dos estudantes em aprender por meio da interação entre estes e o Sistema SWL.

Este artigo foi dividido em 4 seções. Na seção 2, apresenta-se o $i$-collaboration 3.0. Na seção 3, são apresentados a especificação e a implementação do protótipo SWL, seguido da avaliação da solução. A seção 4 apresenta a conclusão do trabalho.

\section{2. i-collaboration 3.0}

Neste trabalho propomos o framework $i$-collaboration 3.0, assim chamado por ser uma extensão ao modelo i-collaboration v1.0 (Oliveira e Tedesco, 2010) e por apoiar a Web Semântica Social ou Web 3.0. A Web 3.0 se preocupa em estudar e viabilizar soluções para as questões de interoperabilidade entre sistemas e a integração de dados distribuídos. O i-collaboration 3.0 busca garantir acesso descentralizado (distribuído) a conteúdos de aprendizado de cursos virtuais disponíveis em AVA e em diversos outros ambientes Web (Twitter, MSN, Blogs, Facebook, Orkut, entre outros), considerando ainda a adaptação e a personalização deste conteúdo, com base nos gostos particulares de cada estudante, através do uso de contexto.

O Quadro 5-1 apresenta a evolução do i-collaboration 1.0 para o i-collaboration 3.0.

Quadro 5-1. Comparativo entre as versões do i-collaboration

\begin{tabular}{|c|c|}
\hline i-collaboration (1.0) & i-collaboration 3.0 \\
\hline Modelo de colaboração inteligente & $\begin{array}{l}\text { Framework de apoio ao desenvolvimento de } \\
\text { AVA distribuídos e sensíveis ao contexto }\end{array}$ \\
\hline Foi utilizado apenas no AVA Cleverpal & $\begin{array}{l}\text { Pode ser integrado e utilizado a partir de } \\
\text { qualquer AVA ou ambiente Web. Não } \\
\text { possui dependências }\end{array}$ \\
\hline $\begin{array}{l}\text { Possui uma instância de agente inteligente } \\
\text { para cada ambiente Web (AVA, Twitter, } \\
\text { MSN, Gtalk, blogs e Websites). Os estudantes } \\
\text { que interagem com o agente inteligente do } \\
\text { modelo em um AVA, não interagem com o } \\
\text { mesmo agente inteligente no Twitter, por } \\
\text { exemplo. Para cada ambiente Web existe um } \\
\text { agente inteligente distinto. }\end{array}$ & $\begin{array}{l}\text { Possui uma única instância do agente } \\
\text { inteligente disponível para todas as } \\
\text { ferramentas Web (AVA, Twitter, MSN, } \\
\text { Gtalk, blogs e Websites). Os estudantes que } \\
\text { iniciam um diálogo com o agente inteligente } \\
\text { do framework através do Twitter, interagem } \\
\text { com o mesmo agente inteligente disponível } \\
\text { no MSN, Gtalk ou quaisquer ambientes } \\
\text { Web. Existe apenas um agente inteligente no } \\
\text { framework e este funciona em diversos } \\
\text { ambientes Web. }\end{array}$ \\
\hline
\end{tabular}




\begin{tabular}{|l|l|}
\hline $\begin{array}{l}\text { As informações contextuais dos estudantes } \\
\text { (conhecimento que cada estudante possui, } \\
\text { perfil MBTI, conteúdo já estudado por cada } \\
\text { estudante, que conteúdos cada estudante } \\
\text { precisa estudar mais) não são integradas entre } \\
\text { os ambientes Web que suportam o modelo }\end{array}$ & $\begin{array}{l}\text { As informações contextuais dos estudantes } \\
\text { Web (AVA, redes sociais, comunicadores } \\
\text { instantâneos) }\end{array}$ \\
\hline $\begin{array}{l}\text { O conteúdo de domínio é personalizado e } \\
\text { distribuído, porém não é integrado }\end{array}$ & $\begin{array}{l}\text { O conteúdo de domínio do sistema é } \\
\text { personalizado, adaptado, distribuído e } \\
\text { consolidado em uma única base de dados }\end{array}$ \\
\hline
\end{tabular}

Para alcançar os objetivos propostos, diversos refinamentos em relação a versão 1.0 do modelo tiveram que ser realizados, conforme apresentados a seguir.

\subsection{Como funciona o i-collaboration 3.0?}

Como forma de facilitar o entendimento sobre o framework proposto, neste trabalho adotamos como exemplo um cenário de uso do i-collaboration 3.0 composto por um estudante, Estudante 1, que se comunica com o agente inteligente inserido no $i$ collaboration 3.0 para aprender os conteúdos de seu curso virtual. Os conteúdos do curso virtual estão disponíveis por meio do uso do i-collaboration $3.0 \mathrm{em}$ diferentes ambientes Web (Figura 1). Neste cenário, o framework de colaboração inteligente está integrado com o Twitter, com o MSN, com alguns sites, com o Facebook e com o Moodle.

No exemplo apresentado, o estudante inicia um diálogo com o agente inteligente do i-collaboration 3.0, por meio do Gtalk, para questioná-lo sobre a função main de um programa em $\mathrm{C}$, tema de estudo em seu curso virtual. $\mathrm{O}$ estudante poderia ter iniciado esta interação com o agente inteligente a partir de qualquer ambiente Web que desejasse. Pouco tempo depois, o mesmo estudante manifesta interesse sobre o mesmo tema de estudo (a função main de um programa C) através de uma comunicação realizada via MSN (Figura 1- passo 3). O agente inteligente reconhece que a pergunta feita nos diferentes ambientes foi feita pelo mesmo estudante e tenta descobrir, por meio de sua base de conhecimentos e considerando a nova pergunta, se o estudante está com algum tipo de dificuldade (análise inteligente e contextualizada de dados distribuídos). O estudante, a partir de qualquer ambiente que faça uso do framework, indica seus diferentes logins nos diversos ambientes Web para o agente inteligente, por meio do uso de (\#) comandos nos chats. 


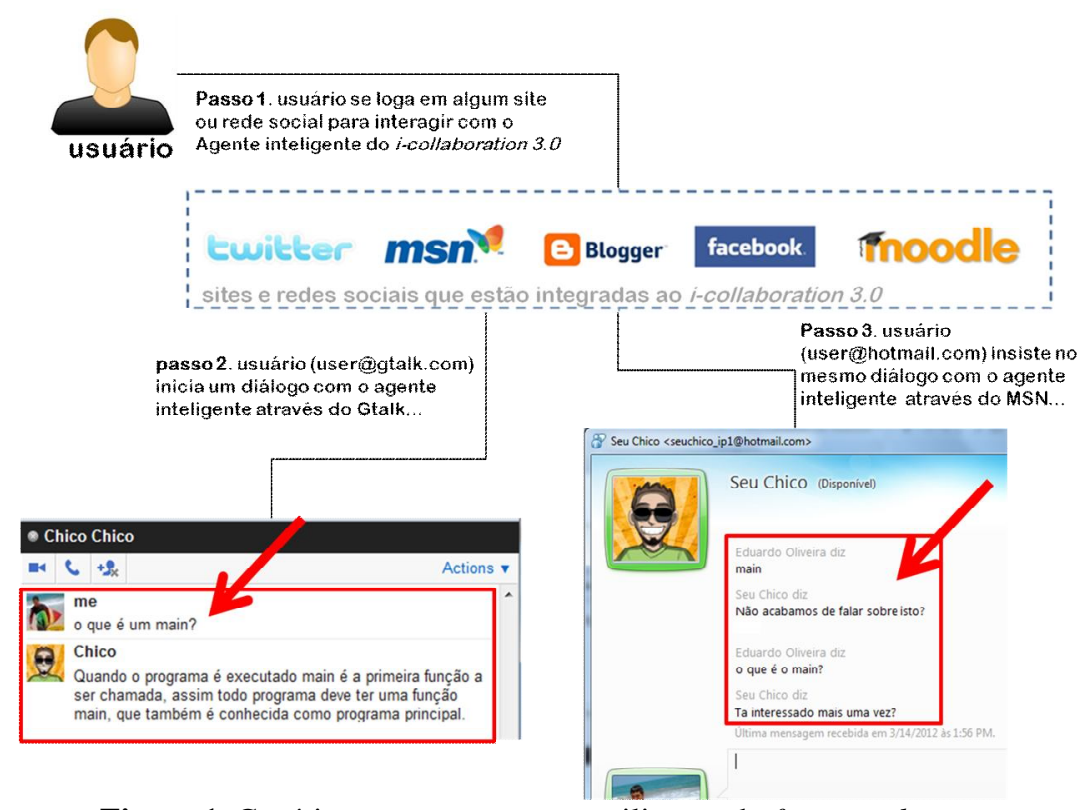

Figura 1. Cenário que apresenta uma utilização do framework proposto

Apesar do chatterbot aparecer em diversos ambientes distintos, o framework provê um único chatterbot compartilhado entre estes ambientes, o que facilita a manutenção dos dados do estudante e a coerência do diálogo. O estudante conversa através de diferentes ambientes com o mesmo agente inteligente. A vantagem em prover um único agente inteligente no framework está no fato de que assim podemos ter também uma única base de dados, compartilhada pelos diversos ambientes distribuídos. Com uma única base de dados, o Estudante 1, que se comunica com o agente inteligente por meio do Gtalk e do MSN pode ser agora identificado nestes ou em qualquer outro ambiente que o agente inteligente esteja presente (ambiente Web integrado com o i-collaboration 3.0). Caso o Estudante 1 demonstre interesse em estudar orientação a objetos, por exemplo, interagindo com o agente inteligente por meio do MSN e, em outro momento, simplesmente solicite indicações de livros ao agente inteligente através de outro ambiente qualquer (Facebook, Twitter, um AVA ou um site), o agente inteligente identificará, por meio de consultas a dados históricos deste estudante, que ele deseja obter indicações de livros de orientação a objetos.

No i-collaboration 3.0, o agente inteligente pode iniciar uma comunicação com o estudante utilizando preferencialmente o ambiente mais utilizado por este. Nesta situação, o agente inteligente pode: recomendar links relevantes ao estudante, alinhados às suas necessidades; sugerir a leitura de conteúdos e a realização de exames; encorajar o estudante em busca de melhores resultados nos estudos; e, recomendar um estudante a outro. Estas ações são tomadas com base no contexto em que se encontram os estudantes (após os estudantes perguntarem sobre determinado conteúdo do curso virtual, após a realização de um exame ou após longo período de inatividade, outros).

Os dados dos estudantes são coletados e analisados com base em sua interação com o agente inteligente do i-collaboration 3.0, de forma dinâmica. A arquitetura desenvolvida para atingir os objetivos do $i$-collaboration 3.0 foi definida com base nos requisitos abaixo:

Requisito 1 - Descentralização no acesso aos conteúdos de cursos virtuais 
No $i$-collaboration 3.0, os ambientes Web e as redes sociais que fazem uso do framework são identificados como clientes do framework. O cliente (ambiente Web ou rede social) que desejar se integrar e utilizar o $i$-collaboration 3.0 deve implementar uma interface provida pelo framework proposto. Após a interface de comunicação do framework ser implementada por um ou mais ambientes Web, uma instância do framework, que conta com o agente inteligente do i-collaboration 3.0, o módulo de contexto e o módulo de testes de personalidade (Myers-Briggs Type Indicator), serão disponibilizados e integrados ao ambiente Web ou rede social, podendo então serem utilizados pelos estudantes.

Requisito 2 - Interoperabilidade

Interoperabilidade é a capacidade de um sistema (informatizado ou não) de se comunicar de forma transparente (ou o mais próximo disso) com outro sistema (semelhante ou não).

Após termos identificado uma forma de permitir a interoperabilidade entre diversos sistemas e ambientes Web com o framework proposto, por meio do uso do agente inteligente, comum a todos os ambientes, nosso terceiro desafio na concepção do framework foi o de garantir a consolidação entre os diversos dados dos estudantes.

Requisito 3 - Consolidação dos dados distribuídos dos estudantes

O i-collaboration 3.0 foi modelado para funcionar com um único agente inteligente e com uma única base de dados. Esta base de dados única é compartilhada por todos os ambientes Web e redes sociais que utilizam o framework. A base de dados guarda informações como o id do estudante, os ambientes Web e redes sociais que este utiliza para aprender, áreas de interesse, resultados de exames realizados, entre outras informações. Quem faz o controle dos dados dos estudantes na base de dados única do framework é o agente inteligente disponível neste.

\section{Requisito 4 - Personalização e Adaptação de Conteúdos}

Um módulo de contexto representa as informações contextuais que devem ser consideradas no framework e como essas informações se relacionam. Com base nesta análise, são realizadas personalizações de conteúdos de aprendizagem com base nos perfis MBTI e comportamentos dos estudantes.

\section{Especificação, implementação do protótipo e avaliação da solução}

Como forma de testar o framework proposto e validar seus conceitos associados (personalização, adaptação, interoperabilidade e consolidação de dados distribuídos), foi desenvolvido o sistema SWL, um protótipo que fez uso do framework i-collaboration 3.0 para disseminar conhecimentos da disciplina Introdução a Programação I, da Universidade Católica de Pernambuco. O SWL foi integrado aos comunicadores instantâneos MSN e Gtalk e ao microblogger Twitter. A escolha destes ambientes Web se deu por conta de suas altas popularidades e pelo grande domínio no uso destes por grande parte dos usuários da Internet, bem como pela simplicidade com que seus usuários conseguem interagir entre si. O Facebook não foi inicialmente integrado a este sistema por conta do grande número de diferentes formas de interação disponibilizadas para os usuários (mural, mensagem inbox, comentário e bate-papo). 
Futuramente, esta rede social também fará parte do SWL. Desta forma, o ambiente virtual de aprendizagem criado com o SWL considerou três diferentes ambientes Web.

\section{Avaliação}

Para validarmos a hipótese deste trabalho, foi realizado um experimento durante 2 meses com alunos do primeiro período do curso de ciência da computação da UNICAP. Após o experimento, detalhado a seguir neste capítulo, foi realizada uma análise sobre os $\log s$ de interação dos estudantes com o agente inteligente provido no SWL, sobre o aprendizado destes e sobre as respostas dos estudantes ao questionário aplicado. Adicionalmente, através da análise dos $\log s$ gerados pelo sistema SWL, uma avaliação quanto à corretude dos conteúdos personalizados e adaptados, em acordo com os perfis dos estudantes, também foi realizada.

\section{Metodologia}

Utilizamos a abordagem Goal/Question/Metric (GQM) (Solingen e Berghout, 1999) como apoio para a organização e a elaboração do experimento.

Neste trabalho, a definição dos objetivos (Goals) foi organizada da seguinte maneira:

- Objetivo global

Identificar se os estudantes se sentirão incentivados a interagir com o Sistema SWL, desenvolvido sobre o framework i-collaboration 3.0, para buscar conteúdos de seus cursos virtuais.

- Objetivo da medição

1. Medir a interação dos estudantes e o acesso a conteúdos providos pelo SWL.

2. Identificar como os estudantes avaliam a corretude dos conteúdos personalizados providos nos diversos ambientes que foram integrados ao SWL.

As questões e métricas (Questions / Metrics) foram organizadas da seguinte maneira:

- Questão 1: Houve grande interação entre os estudantes e o acesso a conteúdos do curso virtual provido no SWL?

- Métrica: Análise de logs de acesso e participação.

- Questão 2: Qual a corretude das mensagens personalizadas e adaptadas entre os ambientes Web distribuídos que são providas aos estudantes, com base nos perfis destes?

- Métrica: A corretude das mensagens fornecidas aos estudantes com base em seus perfis e necessidades e, ambientes utilizados para aprender.

\section{População e Amostra}

A população do experimento foi composta por 65 alunos da disciplina de Introdução a Programação I do Curso de Ciência da Computação da UNICAP. Estes alunos foram escolhidos por utilizarem frequentemente o MSN, o GTalk e o microblogger Twitter.

Dos 65 alunos existentes na população, 35 alunos foram convidados a utilizar o SWL. Esta população foi dividida com a estratégia de podermos comparar os resultados em sala dos estudantes que utilizaram o SWL com os resultados dos demais estudantes. Os 65 estudantes cursaram a disciplina de Introdução a Programação I no turno da noite da Universidade Católica de Pernambuco, e pertenciam a uma mesma sala de aula. Os conteúdos e atividades propostas em sala eram semelhantes a todos os estudantes da disciplina, não existindo distinção entre os que utilizariam o SWL. Deste universo 
escolhido, todos os estudantes concordaram em participar do experimento, colaborando com a realização desta pesquisa.

Para o experimento, decidiu-se não realizar pré-testes para identificar o estado atual dos estudantes antes de iniciar a intervenção (uso do SWL), uma vez que, por meio de entrevistas, identificou-se que nenhum dos estudantes participantes possuía experiência prévia com programação. Todos estavam cursando pela primeira vez a disciplina de Introdução a Programação I.

Os estudantes que não fizeram uso do SWL utilizaram apenas os conteúdos providos em sala de aula, a exemplo de: PPTs, listas de exercícios e indicação de livros. Estes estudantes não tiveram o auxílio de outras ferramentas ou ambientes educacionais. Fizeram uso dos materiais didáticos comumente utilizados na disciplina e compartilhados pelos demais estudantes.

\section{Tempo de Realização do Experimento}

O experimento ocorreu durante dois meses (dias corridos). Durante este período, todas as interações realizadas entre os estudantes e o agente inteligente do sistema SWL (que foi desenvolvido com o apoio do i-collaboration 3.0) foram gravadas para análise futura. Os ambientes integrados com o $i$-collaboration 3.0 foram monitorados durante todos os dias, como forma de garantir que o ambiente se encontraria disponível para utilização $24 \mathrm{~h}$ por dia, 7 dias por semana.

Tarefa

O objetivo da tarefa - utilização do Sistema SWL - proposta ao grupo de estudantes participantes do experimento, foi a de investigar o aumento na interação destes a partir de um ambiente virtual de aprendizagem distribuído e sensível ao contexto. Também foi investigada a corretude na personalização e adaptação dos conteúdos providos neste ambiente desenvolvido.

Foram enviados e-mails com convite de participação na pesquisa e com orientações para a realização da tarefa a todos os estudantes que aceitaram participar. Para a realização da tarefa proposta, foi solicitado aos estudantes a realização do teste de personalidades MBTI e a interação com o agente inteligente do SWL por meio de pelo menos dois dos ambientes Web integrados ao sistema.

Após o período de uso do Sistema SWL, definido em dois meses, foi apresentado aos participantes o questionário da pesquisa. Cada uma das questões (09) permitiu registrar quatro variações (graus de intensidade) para as respostas apresentadas: "Concordo Fortemente", "Concordo", "Discordo" e "Discordo Fortemente". Adicionalmente foi inserida uma última opção - "Neutro", para captura dos participantes que não desejassem expressar sua opinião, possibilidade viável de ocorrer.

\section{Coleta e Análise dos Dados}

Nesta pesquisa, os dados coletados foram provenientes da análise quantitativa das respostas ao questionário proposto aos estudantes participantes do experimento e também pela análise aos $\log s$ do Sistema SWL. O questionário foi aplicado após o período de utilização do Sistema SWL. Os logs consideraram os dados produzidos pelos estudantes ao longo do experimento. Nos logs, informações como login de cada estudante nos diferentes ambientes Web integrados ao SWL, perfil MBTI do estudante, conteúdos estudados, frequência na interação entre o estudante e o agente inteligente do SWL, dia e horário de cada interação realizada com o agente inteligente do SWL, informações quanto a integração de ambientes providos no SWL, entre outras eram armazenadas para análises futuras. Os dados dos estudantes eram armazenados nos logs 
do SWL sempre que um estudante se comunicava com o agente inteligente do SWL, fosse esta comunicação realizada através de comandos (\#) ou de linguagem natural.

Para a análise das informações coletadas utilizamos em apoio o software Microsoft Excel 2007. Dos 35 estudantes convidados a utilizar o SWL, 29 interagiram com o agente inteligente disponível no Sistema SWL. Os demais optaram por não utilizar o sistema, por decisões pessoais. Os estudantes foram instruídos e recomendados a responder o teste de personalidades MBTI provido no Sistema SWL, contudo esta escolha não era obrigatória e não inviabilizava o acesso destes ao ambiente educacional. O teste de personalidades MBTI serve como entrada para o agente inteligente do Sistema SWL lidar de maneira diferenciada com cada estudante, baseado em seu perfil. Por meio do MBTI, o agente inteligente decide quanto a melhor maneira de interagir com cada estudante. Entre os estudantes participantes, apenas 18 responderam ao teste de personalidades, tendo estes sido classificados entre 8 dos 16 tipos de personalidade suportados. Para cada personalidade de estudante observada, validamos, por meio de análises ao $\log$ do Sistema SWL, que cem por cento (100\%) das mensagens providas pelo agente inteligente do SWL corresponderam aos diferentes perfis observados.

Mais de 800 interações, realizadas entre os estudantes que responderam ao teste de personalidades MBTI e o agente inteligente do Sistema SWL, no MSN, no Gtalk e no Twitter, foram observadas e validadas. Esta verificação foi realizada de maneira manual. Os 11 estudantes que não responderam ao teste de personalidades foram verificados da mesma maneira, em um perfil geral, sem personalização de conteúdos. Por meio de análises ao log, identificamos se os conteúdos providos a partir de suas interações foram apropriados ao perfil default.

Após analisar e validar a personalização de conteúdos no Sistema SWL, analisamos a interoperabilidade provida entre os ambientes educacionais disponibilizados. Neste caso, estávamos interessados em identificar se um mesmo estudante que interagiu com o agente inteligente do Sistema SWL por meio do MSN, do Gtalk e do Twitter era identificado unicamente. Apesar das instruções, 11 estudantes optaram por interoperacionalizar seus ambientes e consolidar seus dados distribuídos entre o MSN, o Gtalk e o Twitter. Conforme análise realizada nos logs do sistema, estes 11 estudantes também responderam ao teste de personalidades MBTI, tendo estes utilizado todas as funcionalidades do sistema.

Através da análise de $\log s$ e do banco de dados do sistema, validamos as informações de todos os estudantes que integraram seus ambientes. Verificamos cada interação realizada por cada um destes estudantes e se o agente os identificou corretamente de maneira distribuída. 100\% (cem por cento) dos casos investigados consideraram o contexto de uso dos estudantes. Para chegar a esta conclusão e a este número, todos os logs do sistema foram avaliados de maneira manual. Para cada um dos 11 estudantes que integraram seus ambientes, todas as interações foram validadas.

Por fim, os questionários que foram aplicados após o experimento com os estudantes que utilizaram o SWL, foram avaliados. Dos 29 estudantes que utilizaram o Sistema SWL, 25 responderam ao questionário.

Após analisar cada um dos itens do questionário, verificamos que para os estudantes, o Sistema SWL auxiliou no aprendizado da disciplina Introdução a Programação I e que se apresentou de maneira fácil em seu uso. Todos os estudantes que responderam ao questionário afirmaram conhecer os ambientes Web integrados ao SWL (MSN, Gtalk e Twitter). Apesar de apenas 8 estudantes afirmarem ter tido 
experiências prévias com ambientes educacionais virtuais, isto não determinou o sucesso no entendimento e uso do Sistema SWL. Para a grande maioria dos estudantes, as mensagens providas pelo agente inteligente do sistema foram motivadoras e despertaram interesse. Apesar de parte dos estudantes ter afirmado que não teve participação grande durante todo o experimento, a grande maioria afirmou que conseguiu se comunicar com o agente inteligente do sistema.

Os resultados do questionário e das análises de $l o g$, dos dados do banco de dados e das comparações realizadas após a primeira avaliação realizada na disciplina indicam que o Sistema SWL atendeu às expectativas dos estudantes. Após 2 meses de utilização do sistema, observamos o satisfatório interesse dos estudantes em aprender por meio da interação entre estes e o Sistema SWL

\section{Conclusão}

Os AVA permitem aos estudantes uma nova forma de aprendizado. Aprender virtualmente precisa ser encarado como uma atividade motivadora, onde os estudantes, sujeitos ativos em seu processo de aprendizado, utilizam-se da flexibilidade de tempo e de espaço para buscar conhecimento sob as mais diversas formas digitais disponíveis. Neste contexto, o uso de AVA distribuídos e sensíveis ao contexto apresentam-se como diferencial para tornar o aprendizado virtual mais interessante.

Através da proposta do i-collaboration 3.0, neste trabalho apresentou-se uma contribuição para os problemas de desmotivação que tem tornado difícil o uso dos AVA por seus participantes, dando-lhes um incentivo a mais no processo educativo. Buscou-se ainda oferecer aos estudantes uma condição mais social no meio virtual, fornecendo-lhes distribuição e personalização de conteúdos educacionais a partir de diversos ambientes Web que já estão acostumados a utilizar.

Os resultados obtidos através do experimento realizado sugerem que a aplicação do framework no Sistema SWL pode contribuir para o maior interesse dos estudantes na busca do conhecimento. O framework será mais testado e utilizado como forma de assegurar a qualidade dos resultados obtidos até o momento.

\section{Referências}

MEYROWITZ, J., Global Nomads in the digital veldt., in Revista Famecos, julho, 2004, PUC-RS, Porto Alegre, pp. 23-30.

MYERS \& BRIGGS FOUNDATION: About the MBTI Instrument. Disponível em: http://www.myersbriggs.org. Acesso em: 04 jun, 2013.

OLIVEIRA, E. A. ; TEDESCO, P. i-collaboration: Um modelo de colaboração inteligente personalizada para ambientes de EAD. Revista Brasileira de Informática na Educação, v. 18, p. 17-31, 2010.

SOLINGEN, R., BERGHOUT, E., "The Goal/Question/Metric Method: a Practical Guide for Quality Improvement of Software Development", the McGraw-Hill Companies, UK, 1999.

VIEIRA, V., SOUZA D., SALGADO, A. C., TEDESCO, P. (2006) "Uso e Representação de Contexto em Sistemas Computacionais". In: Cesar A. C. Teixeira; Clever Ricardo G. de Farias; Jair C. Leite; Raquel O. Prates. (Org.). Tópicos em Sistemas Interativos e Colaborativos. São Carlos: UFSCAR, 2006, v. , p. 127-166. 